\title{
A prospective comparison of subjective and objective assessments of cosmetic outcomes following breast brachytherapy
}

\author{
Tabassum Wadasadawala, MD', Shwetabh Sinha, MD², Shalini Verma, MD², Dr Vani Parmar, MS³, Sadhana Kannan, MSc', \\ Rima Pathak, MD², Rajiv Sarin, MD, FRCR', Mithila Gaikar, BSc ${ }^{5}$ \\ 'Department of Radiation Oncology, Advanced Centre for Treatment, Research and Education in Cancer, Tata Memorial Centre, Homi \\ Bhabha National Institute, Kharghar, Navi Mumbai, India, ${ }^{2}$ Department of Radiation Oncology, Tata Memorial Centre, Homi Bhabha \\ National Institute, Parel, Mumbai, India, ${ }^{3}$ Department of Surgical Oncology. Advanced Centre for Treatment, Research and Education in \\ Cancer, Tata Memorial Centre, Homi Bhabha National Institute, Kharghar, Navi Mumbai, India, ${ }^{4}$ Department of Biostatistics, Advanced Centre \\ for Treatment, Research and Education in Cancer, Tata Memorial Centre, Homi Bhabha National Institute, Kharghar, Navi Mumbai, India, \\ ${ }^{5}$ Clinical Research Secretariat, Tata Memorial Centre, Homi Bhabha National Institute, Parel, Mumbai, India
}

\begin{abstract}
Purpose: We evaluated agreement between subjective and objective methods of cosmesis scoring in an accelerated partial breast irradiation (APBI) cohort.

Material and methods: Consecutive women treated with APBI using interstitial brachytherapy reported for clinical follow-up every 6 months. Single cross-sectional assessment of the breast cosmesis was done by a radiation oncologist (subjective method) using Harvard scale and by photographic assessment using BCCT.core (Breast Cancer Conservative Treatment. Cosmetic results, version 3.1) software (objective method) at 18-36 months post-APBI. The agreement between subjective and objective methods for the overall score as well as individual subjective/objective subdomains was computed using kappa statistics. ANOVA was used to test the correlation between objective indices and subjective subdomains.

Results: The agreement between the subjective (physician) and objective assessment was good with a kappa of 0.673. Overall, $77(98.7 \%)$ patients were satisfied with the overall outcomes of breast conservation therapy. The kappa agreement between physician and patient scoring was 0.457 (95\% CI: 0.240-0.674). Among the subjective subdomains, location of the nipple areola complex (NAC) had good agreement with both the overall subjective and objective score, with the kappa of 0.778 and 0.547 , respectively. In the objective indices, BCE (breast compliance evaluation), LBC (lower breast contour), and UNR (unilateral nipple retraction) correlated significantly with the subjective subdomains: location of the NAC, breast size, and shape ( $p<0.05$ for all indices).

Conclusions: Good agreement exists for overall cosmetic outcomes measured by subjective and objective methods. Location of the NAC, breast size and shape are the most important parameters determining cosmetic outcomes irrespective of the method of assessment.

J Contemp Brachytherapy 2019; 11, 3: 207-214 DOI: http://dx.doi.org/10.5114/jcb.2019.85414
\end{abstract}

Key words: APBI, brachytherapy, cosmesis, BCCT.core.

\section{Purpose}

Accelerated partial breast irradiation (APBI) is now one of the standards of care for suitably selected women with node-negative early breast cancers [1,2,3,4]. Besides offering the patients and physicians an opportunity for reducing the overall treatment time, it may be beneficial in terms of cost, toxicity and cosmetic outcome $[5,6]$. Interstitial brachytherapy with multichannel nylon catheters remains one of the most commonly used strategies for APBI, especially in low- and middle-income countries [7].

Cosmesis after APBI remains an important endpoint. Many methods have been described in the literature for assessing cosmesis after breast conservation therapy (BCT) $[8,9]$. These include subjective methods like the visual assessment of post-treatment breast and its comparison with the unaffected breast, scored by the patient, the physician, or a trained nurse. This can be evaluated either using a 4-point Harris scale or a 2-point Pezner 
scale $[10,11]$. Objective methods include photographic assessment of baseline and the post-treatment photographs [12]. High inter-user and intra-user variability in the subjective assessment of cosmesis lead to the introduction of objective methods of cosmetic assessment. Initially, various aspects of breast cosmesis like the size, shape, symmetry, ptosis, and the location of nipple were studied individually $[13,14,15,16,17,18]$. However recently, the BCCT.core software (Breast Cancer Conservative Treatment. Cosmetic results 3.1) is being increasingly used in the objective assessment of breast cosmesis because it provides a composite score of breast symmetry and also takes into account the colour and scar appearance [19]. The BCCT.core software utilizes seven breast asymmetry parameters to give an overall rating on a 4-point Likert scale ('excellent', 'good', 'fair', 'poor'). The parameters include breast retraction assessment (BRA), lower breast contour (LBC), upward nipple retraction (UNR), breast compliance evaluation (BCE), breast contour difference $(\mathrm{BCD})$, breast area difference (BAD), and breast overlap difference (BOD) [20]. However, so far, no gold standard has been established, and most oncologists favour incorporation of both subjective and objective methods for assessment of cosmesis [21].

In a prospective cross-sectional study, we evaluated the breast cosmesis after APBI delivery using interstitial brachytherapy by both subjective (assessment by radiation oncologist) and objective (BCCT.core 3.1) methods and correlated the subjective subdomains with the overall objective and subjective cosmetic scores. We also studied the correlation of various objective indices derived from BCCT.core with the subjective subdomains, as this has not been evaluated earlier. The results of this study help us understand the clinical relevance of these quantitative measures in the evaluation of breast cosmesis.

\section{Material and methods}

Seventy-eight patients who had undergone APBI with interstitial brachytherapy using multichannel nylon catheters were prospectively enrolled in this study. Breast conservation surgery with wide margins (>5 mm) was performed by experienced breast surgeons. All surgeries were completed by open cavity technique and interstitial implant was done using freehand method. Patients underwent local treatment and appropriate systemic therapy from January 2015 to November 2016. Subsequently, patients were called for clinical evaluation every 6 months up to 5 years post-treatment and annually thereafter. APBI was performed either intraoperatively or postoperatively using nylon catheters (flexible implant nylon tubes, Kalyani Enterprises Inc.). 3-dimensional conformal treatment planning was completed with Oncentra treatment planning system (version 4.3; Nucletron, Elekta, Stockholm, Sweden). Tumor bed was delineated on planning CT images taking into account the seroma cavity, post-operative changes, and tumor bed clips. A margin of 1.0-1.5 cm was given to the tumor bed to delineate clinical target volume (CTV). The CTV was edited $5 \mathrm{~mm}$ from the skin and posteriorly from the chest wall. The details of the method of implant and dosimetry from our institute have been published earlier [22]. The dose prescribed to the CTV was $34 \mathrm{~Gy}$ in 10 fractions or 32 Gy in 8 fractions, delivered twice daily, 6 hours apart. Brachytherapy was initiated on the day of the implant in post-operative patients and after 3 days in the intraoperative group. A gap of 2-3 weeks was mandatory between the brachytherapy procedure and the first (intraop cases) or last (post-op cases) cycle of chemotherapy in order to prevent post-procedure complications.

The study lasted from January 2017 to June 2018, and it was conducted after institutional review board approval and a written informed consent obtained from all participants. Cosmesis was assessed for all patients in a one-time cross-sectional manner, between 18 to 36 months after completion of APBI. At the time of routine clinical follow-up, all patients underwent clinical examination and toxicity assessment in the outpatient clinic. Eligible patients were counselled regarding the study and patients who consented for the photographic assessment were accrued. Breast photographs were taken in standing position with arms overhead and by the side, using a 16-megapixel $4 \times$ optical zoom camera. Prior to obtaining the photographs, it was mandatory to put two marks on the patient's skin using a marker pen to serve as a reference scale for calculation of indices in the BCCT.core software. One mark was put on the sternal notch and the other $25 \mathrm{~cm}$ inferior from the sternal mark. The photographs were taken in a well-lit room from a distance of 1 meter. The user license agreement for the BCCT.core software was obtained from INESC Porto breast research group prior to the start of the study. All patients filled the EORTC QLQ C30 and BR23 quality of life forms and also scored their own cosmesis and satisfaction with breast conservation.

Subjective cosmesis assessment was evaluated by a single experienced radiation oncologist $(\mathrm{RO})$ using the Harvard 4-point scale for overall cosmesis, with 'excellent', 'good', 'fair', and 'poor' scores. Individual subdomains affecting cosmesis were also assessed on a 4-point scale ('no difference', 'little difference', 'moderate difference', and 'large difference'), which included the breast size, shape, colour, scar appearance, shape of nipple-areola complex (NAC), and the location of the NAC.

All the 4-point scale, the variables were dichotomized to a 2-point scale for the purpose of improving agreement as has been described in the literature. 'Excellent' and 'good' cosmesis were clubbed together as a single group 'excellent/good' (EG), while 'fair' and 'poor' cosmesis were grouped in a single group as 'fair/poor' (FP). Similarly, the subjective subdomains were also dichotomized: subjective size, shape, colour, location of the NAC, and the shape of NAC were categorized as 'no difference/ small difference' vs. 'moderate difference/large difference', while the appearance of scar was categorized as 'very unobtrusive/visible but not affecting cosmesis' vs. 'visible and affecting cosmesis somewhat/visible and affecting cosmesis significantly'.

For statistical analysis, descriptive statistics were used to assess the overall subjective and objective scores as well as the patient rating of the cosmesis and satisfaction. Cohen's kappa statistics was used to determine the 
agreement between subjective and objective cosmesis, and between individual subjective parameters and overall subjective/objective cosmesis. One-way ANOVA was used to assess the agreement between individual indices (continuous variables) obtained from BCCT.core and the subjective subdomains (categorical variables).

The raw scores of various quality of life (QoL) parameters were converted into scale scores out of 100 using linear transformation for analysis as per guidelines from the EORTC [23]. The mean, median, and standard deviations of the functional and symptom scales were reported both for QLQ C30 and BR23. All the analysis was completed in SPSS, version 21.0 (IBM Corp. released 2012. IBM SPSS Statistics for Windows, version 21.0. Armonk, NY, IBM Corp.).

\section{Results}

The median age for the entire cohort was 51 years (range, 44-82 years). Sixty-six (84.6\%) women were post-menopausal, while $12(15.3 \%)$ were peri-menopaus$\mathrm{al} /$ pre-menopausal. Ten $(12.8 \%)$ patients had a history of diabetes mellitus, while $2(2.5 \%)$ women had a history of chronic smoking. Tumor was located in upper outer quadrant (UOQ) in 27 (34.6\%) patients, upper inner quadrant (UIQ) in $26(33.3 \%)$, lower outer quadrant (LOQ) in $3(3.8 \%)$, lower inner quadrant (LIQ) in $9(11.5 \%)$, and centrally in $13(16.6 \%)$ of patients.
The median volume of the CTV was $100.6 \mathrm{cc}$ (range, 14-300 cc). The median number of tubes and planes was 20 and 3 , respectively. The median coverage index (CI) for the tumor bed and CTV were 0.95 and 0.81 , respectively. The median conformity index (COIN) and dose homogeneity index (DHI) were 0.71 and 0.70 , respectively. The median time between start (post-op cases) or end (intraop cases) of brachytherapy and initiation of chemotherapy was 24 days (range, 21-41 days).

A total of 78 patients were enrolled and assessed. Overall cosmesis assessment by RO was EG for 53 patients and FP for 25 patients, whereas 54 EG and 24 FP for assessment by BCCT, as shown in Table 1. The kappa agreement between $\mathrm{RO}$ assessment and objective assessment was 0.673 (95\% CI: 0.495-0.850). Figure 1 shows patient's photographs for EG and FP cosmesis.

Sixty-six (84.6\%) patients rated their cosmesis as EG and $12(15.4 \%)$ patients rated their cosmesis as FP. Despite this, $77(98.7 \%)$ patients were satisfied with the overall outcomes of brachytherapy. The kappa agreement between physician and patient scoring was 0.457 (95\% CI: 0.240-0.674).

Among the subjective subdomains, the size of breast, shape of breast, location of NAC, shape of NAC, and the appearance of scar were correlated with objective and subjective cosmesis. Color of the breast was not analyzed for agreement, with overall cosmetic scores as 77 (98.7\%) patients had no difference/small difference in the colour of skin (Table 2).

Table 1. Agreement between cosmesis assessed by subjective (physician and patient) and objective methods

\begin{tabular}{|c|c|c|c|c|c|c|}
\hline & & \multicolumn{2}{|c|}{ Objective assessment (BCCT.core) } & & \multirow[t]{2}{*}{ Kappa } & \multirow[t]{2}{*}{ Standard error $(95 \% \mathrm{CI})$} \\
\hline & & EG & FP & & & \\
\hline \multirow{2}{*}{$\begin{array}{l}\text { Physician } \\
\text { assessment }\end{array}$} & EG & 48 & 5 & 53 & \multirow[t]{3}{*}{0.673} & \multirow[t]{3}{*}{$0.091(0.495-0.850)$} \\
\hline & FP & 6 & 19 & 25 & & \\
\hline Total & & 54 & 24 & 78 & & \\
\hline \multirow{2}{*}{$\begin{array}{l}\text { Patient } \\
\text { assessment }\end{array}$} & $\mathrm{EG}$ & 48 & 18 & 66 & \multirow[t]{3}{*}{0.161} & \multirow[t]{3}{*}{$0.112(0.059-0.381)$} \\
\hline & FP & 6 & 6 & 12 & & \\
\hline Total & & 54 & 24 & 78 & & \\
\hline
\end{tabular}

EG - excellent/good, FP-fair/poor
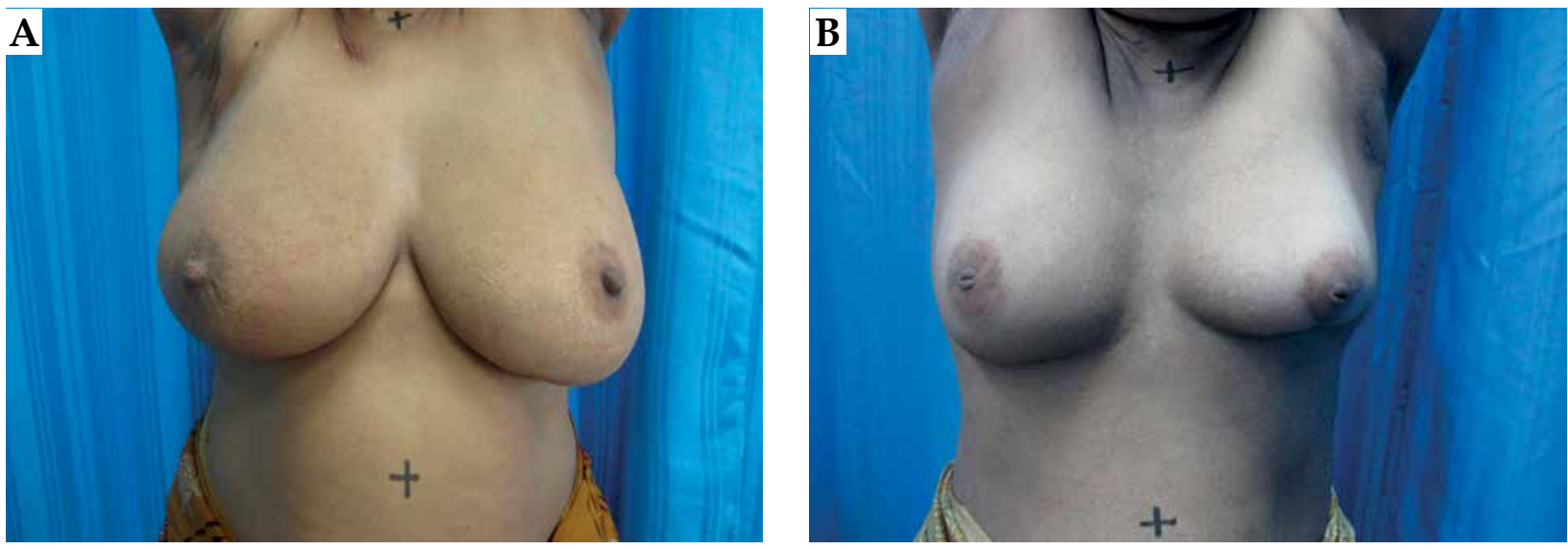

Fig. 1. Excellent/good (EG) and fair/poor (FP) cosmesis 
The agreement of individual subject subdomains with the overall objective score is presented in Table 3, while Table 4 shows the agreement tested using ANOVA between individual objective indices and individual subjective subdomains. The patient-reported outcomes from the analysis of QLQ C30 and BR23 are presented in Table 5.

\section{Discussion}

The basic premise for breast conserving therapy is the superior cosmetic outcome as compared to mastectomy; which leads to a better quality of life and psycho-social well-being $[24,25]$. The current study reports excellent/ good cosmetic outcomes in the majority of patients by all methods of cosmetic assessment. Various subjective and objective methods for cosmesis evaluation have been described in literature, but there is no consensus on standardization of these methods. Recently, many studies have shown that incorporation of both a subjective and objective method may give the best possible measure of patient's cosmetic outcomes [12,21,26]. A combined assessment, although desirable, would be time consuming and logistically challenging in busy setups, and as such may not be practical in everyday clinical practice.

Partial breast irradiation is an attractive treatment option for early breast cancer. However, randomized data with regards to quality of life, cosmesis, and toxicity of APBI is still limited. The RAPID trial reported inferior cosmetic outcomes of 3D-CRT-based APBI as compared to whole breast radiotherapy (WBRT) [27]. The authors attributed the inferior outcomes of 3D-CRT-based APBI to larger amount of normal tissue irradiated. However, more recently published IMPORT LOW and Florence trials have demonstrated non-inferior patient-reported outcomes and quality of life with IMRT-based APBI $[28,29]$. Most of the available literature on APBI is with multichannel interstitial brachytherapy. The long term results of GEC-ESTRO phase III randomized trial of interstitial

Table 2. Agreement of overall subjective cosmesis scores with individual subjective subdomains

\begin{tabular}{lccc} 
Subjective subdomain & Kappa & Standard error $(95 \% \mathrm{Cl})$ & $P$ value \\
\hline Breast size & 0.433 & $0.104(0.229-0.637)$ & 0.021 \\
\hline Breast shape & 0.405 & $0.107(0.197-0.614)$ & 0.0001 \\
\hline Location of NAC & 0.778 & $0.078(0.624-0.931)$ & 0.0001 \\
\hline Shape of NAC & 0.300 & $0.100(0.105-0.496)$ & 0.001 \\
\hline Appearance of scar & 0.368 & $0.104(0.158-0.564)$ & 0.001
\end{tabular}

NAC - nipple areola complex

Table 3. Agreement of overall objective cosmesis scores with individual subjective subdomains

\begin{tabular}{lccc} 
Subjective subdomain & Kappa & Standard error $(95 \% \mathrm{Cl})$ & $P$ value \\
\hline Breast size & 0.235 & $0.109(0.021-0.449)$ & 0.021 \\
\hline Breast shape & 0.210 & $0.110(-0.007-0.426)$ & 0.0001 \\
\hline Location of NAC & 0.547 & $0.105(0.341-0.753)$ & 0.0009 \\
\hline Shape of NAC & 0.088 & $0.093(-0.095-0.270)$ & 0.0007 \\
\hline Appearance of scar & 0.119 & $0.108(-0.093-0.332)$ & 0.328
\end{tabular}

NAC-nipple areola complex

Table 4. Correlation between individual BCCT indices with individual subjective subdomains

\begin{tabular}{lccc} 
BCCT indices and subjective subdomain & Mean & Standard error & $P$ value \\
\hline BRA and location of NAC & 2.80 & 0.181 & 0.004 \\
\hline UNR and location of NAC & 1.97 & 0.196 & 0.001 \\
\hline BCE and location of NAC & 1.28 & 0.136 & 0.001 \\
\hline LBC and location of NAC & 1.35 & 0.107 & 0.001 \\
\hline BCE and breast size & 1.28 & 0.135 & 0.02 \\
\hline BCE and breast shape & 1.31 & 0.135 & 0.001 \\
\hline LBC and breast size & 1.35 & 0.107 & 0.0009 \\
\hline LBC and breast shape & 1.35 & 0.107 & 0.002
\end{tabular}

$B R A$ - breast retraction assessment, NAC - nipple areola complex, UNR - upward nipple retraction, BCE - breast compliance evaluation, $L B C$ - lower breast contour 
Table 5. Patient-reported quality of life using EORTC QLQ C30 and BR23

\begin{tabular}{|c|c|c|c|}
\hline Domain & Mean & Median & Standard deviation \\
\hline Physical functioning & 82.90 & 86.66 & 18.28 \\
\hline Role functioning & 91.23 & 100.00 & 15.59 \\
\hline Emotional functioning & 78.31 & 83.33 & 22.48 \\
\hline Cognitive functioning & 83.54 & 83.33 & 18.31 \\
\hline Social functioning & 94.23 & 100.00 & 11.65 \\
\hline Fatigue & 27.06 & 22.22 & 20.85 \\
\hline Nausea vomiting & 5.34 & 00.00 & 11.23 \\
\hline Pain & 19.44 & 16.66 & 22.37 \\
\hline Dyspnea & 11.53 & 00.00 & 19.24 \\
\hline Sleep & 17.52 & 00.00 & 25.60 \\
\hline Appetite & 13.24 & 00.00 & 23.00 \\
\hline Constipation & 16.23 & 00.00 & 24.46 \\
\hline Diarrhea & 4.70 & 00.00 & 13.93 \\
\hline Financial difficulty & 17.09 & 00.00 & 23.25 \\
\hline Global health score & 73.50 & 79.16 & 19.02 \\
\hline Body image & 93.91 & 100.00 & 13.27 \\
\hline Sexual functioning & 92.52 & 100.00 & 17.74 \\
\hline Sexual enjoyment & 91.88 & 100.00 & 20.23 \\
\hline Future perspective & 76.06 & 66.66 & 27.86 \\
\hline Systemic therapy side effects & 17.94 & 14.28 & 16.79 \\
\hline Breast symptoms & 9.61 & 00.00 & 13.76 \\
\hline Arm symptoms & 16.38 & 11.11 & 18.14 \\
\hline Hair loss & 14.10 & 00.00 & 30.63 \\
\hline
\end{tabular}

brachytherapy APBI and WBRT was published in 2018 [30]. In addition to the fact that APBI was non-inferior to WBRT in terms of quality of life, the breast symptom score at baseline, 2 and 3 months follow-up was superior in APBI arm. All these randomized data suggest that APBI with modern techniques like IMRT or interstitial multichannel catheter results in excellent long-term quality of life and favorable toxicity profile.

The assessment of cosmesis after breast cancer has evolved considerably over time. One of the earliest reports of cosmesis assessment was by Harris et al., which categorized the overall cosmesis as 'excellent', 'good', 'fair', and 'poor' [10]. It was subsequently expanded to include the appearance of surgical scar, breast size, breast shape, skin colour, the location of areola and nipple, and the shape of areola and nipple by Aaronson [25]. We used this scale in the current study and observed correlation of individual scale with the overall subjective and overall objective score. Such an assessment will be beneficial for the surgeon and radiation oncologist in the baseline patient selection and procedural planning for BCT incor- porating interstitial brachytherapy-based APBI. In this study, we used the BCCT.core 3.1 software for objective assessment, as this is one of the tools that is freely available and has been previously validated $[20,31,32]$.

The overall cosmesis was EG for 53 out of 78 patients for subjective assessment $(67.9 \%)$ and 54 out of 78 patients $(69.2 \%)$ by objective criteria. This is in accordance with the other series reported in literature including one from our own institute $[5,21]$. The overall agreement between the physician rating and the objective assessment was $85.8 \%$ with a kappa coefficient of 0.673 (95\% CI: $0.453-0.850)$. However, the agreement of patient scoring and the objective scoring was lower $(69.23 \%)$ with a much lower kappa of 0.161 (95\% CI: 0.059-0.381). Corica et al. similarly reported an agreement rate between $66.7 \%$ to $85.1 \%$ for assessment by the doctor and BCCT.core, and $66.7 \%$ to $74.5 \%$ for assessment by patient and BCCT.core in the TARGIT-A cohort [31]. The kappa score for physician rating was much higher in the current study than that reported by Merie et al. in a large cohort of randomized patients evaluating the impact of breast boost on cos- 
metic outcome [21]. This is because in Merie et al. study, a direct patient-reporting was done, whereas in this study, the photographic rating was used.

The individual parameters that were significantly associated with both inferior subjective and objective outcomes were the size and shape of breast as well as the location of NAC. However, the kappa for location of the NAC showed moderate and consistent agreement with both subjective and objective score. The breast size and shape reflect the volume of tissue removed and the cavity size. In our study, the median cavity size was $98 \mathrm{cc}$ (range, 14-270 cc). Several other authors have found this to be the most important factor for a good cosmesis for breast conserving therapy $[8,28,33]$. The size and shape of the breast may also be affected to a certain extent by radiation-induced late fibrosis. As such, a high tumor to breast ratio remains the most important factor affecting cosmesis, and minimizing the procedures should be considered [34]. Hence, it is important to restrict the volume of breast being irradiated to have good cosmetic outcomes post-APBI. Moreover, it is recommended to follow strict guidelines for ensuring good quality of implant $[1,35]$. For assessment of software using BCCT.core, the markers are placed at the supra-sternal notch, with the reference point ( $25 \mathrm{~cm}$ below the supra-sternal notch) at the level of nipples bilaterally. Therefore, a higher weightage to the location of the NAC in objective assessment is expected. Many other studies have also affirmed that preservation of the NAC is an important factor that determines subjective satisfaction after surgery for breast cancer [36,37]. Therefore, all efforts must be made to preserve the location and shape of the NAC in these early breast cancer women.

Each of the seven BCCT indices was correlated with the five subdomains of the subjective assessment, but only significant results are presented in Table 4. It was observed that BCE and LBC were consistently in agreement with the breast size and shape as well as with the nipple location. In addition, the nipple location correlated strongly with UNR. Authors have not come across any study reporting such associations and probably, this is the first study reporting on these relations.

Scar appearance is of great importance for a woman as shown by Sneeuw et al. [36]. In our series, it was significant only for the subjective overall assessment. This can be attributed to the fact the weightage for scar appearance/prominence may be more in subjective cosmetic assessment as compared to objective scoring. We did not find any significant agreement of colour of the breast with the overall subjective or objective outcomes, although this has been a well-documented risk factor for poor cosmetic outcomes in many series. We attribute this fact to the small volume of breast irradiated in the APBI cohort, with 67 patients out of 78 having no difference in the colour of irradiated breast and 11 patients having only a small difference. On the other hand, external radiation comprising of the whole breast as well as boost is expected to cause pigment changes and an increase of the incidence of telangiectasias. Although such changes are not observed in breast brachytherapy, skin changes at the catheter entries and exit points can impact cosmesis. As the current study was cross sectional in design women who were at least 18 months post-brachytherapy, the skin changes at the catheter points were expected to subside.

Moreover, other factors like a high body mass index (BMI), diabetes mellitus, smoking, and the use of hormonal therapy/chemotherapy are known to adversely impact cosmesis after breast conserving therapy $[14,16,17,38,39]$. In the current cohort, the number of women with a history of smoking and diabetes was low $(2.5 \%$ and $12.8 \%$, respectively), as compared to the western population. Adjuvant hormonal therapy was given to 72 (92.3\%) of women, while $42(53.8 \%)$ received adjuvant chemotherapy. Adjuvant chemotherapy, which starts within 3 weeks of implant is known to have inferior cosmetic outcomes $[40,41]$. In our study, all patients started adjuvant chemotherapy after 3 weeks of implant.

The TARGIT-A trial has reported a cosmetic outcome evaluated over 5 years using the 4-point Harris scale and BCCT.core, but authors have not reported the agreement between the subdomains of both subjective as well as objective methods, as presented in our study. A detailed record of the subjective assessment provided the opportunity to analyze and report these results. Hence, the study results add an important piece of information on the cosmetic evaluation methods for breast cancer.

The limitations of this study include limited sample size, cross-sectional design not allowing temporal evaluation, and lack of assessment by a group or panel. The authors agree that a cross-sectional one-time evaluation may not be the most accurate method for assessing cosmesis, as it can change considerably over time. We chose a one-time cross-sectional design as the primary aim of this study to correlate subjective and objective methods. A time point of 18-36 months post-implant was chosen, as this is the period where maximum late sequalae are likely to have an impact on cosmetic outcome. Moreover, patients are more compliant to follow-up in the early years following completion of treatment. We chose assessment by a single radiation oncologist (with experience in treating breast cancers for more than 10 years) as the method of subjective evaluation to reduce the inter-observer variability.

\section{Conclusions}

We conclude that there is a moderate agreement between subjective and objective methods of cosmesis assessment. Location of the NAC is the most important parameter that impacts cosmesis after brachytherapy along with the shape and size of the breast. Individual indices of objective assessment also correlate strongly with these three parameters.

\section{Acknowledgements}

We would like to thank INESC Porto breast research group for providing us with BCCT.core v. 3.1 software.

\section{Disclosure}

Authors report no conflict of interest. 


\section{References}

1. Shah C, Vicini F, Shaitelman SF et al. The American Brachytherapy Society consensus statement for accelerated partial-breast irradiation. Brachytherapy 2018; 17: 154-170.

2. NCCN Guidelines for Patients ${ }^{\circledR} \mid$ Breast Cancer - Stages I and II. https://www.nccn.org/patients/guidelines/recent updates/breast_stage_i_and_ii.aspx

3. Strnad V, Major T, Polgar C et al. ESTRO-ACROP guideline: Interstitial multi-catheter breast brachytherapy as Accelerated Partial Breast Irradiation alone or as boost - GEC-ESTRO Breast Cancer Working Group practical recommendations. Radiother Oncol 2018; 128: 411-420.

4. Correa C, Harris EE, Leonardi MC et al. Accelerated partial breast irradiation: executive summary for the update of an ASTRO evidence-based consensus statement. Pract Radiat Oncol 2017; 7: 73-79.

5. Wadasadawala T, Sarin R, Budrukkar A et al. Accelerated partial-breast irradiation vs conventional whole-breast radiotherapy in early breast cancer: A case-control study of disease control, cosmesis, and complications. J Cancer Res Ther 2009; 5: 93-101.

6. Harat A, Harat M, Makarewicz R. Whole breast irradiation vs. APBI using multicatheter brachytherapy in early breast cancer - simulation of treatment costs based on phase 3 trial data. J Contemp Brachytherapy 2016; 8: 505-511.

7. Sarin R. Partial-breast treatment for early breast cancer: emergence of a new paradigm. Nat Clin Pract Oncol 2005; 2 . 40-47.

8. Garsa AA, Ferraro DJ, DeWees T et al. Cosmetic analysis following breast-conserving surgery and adjuvant high-doserate interstitial brachytherapy for early-stage breast cancer: a prospective clinical study. Int J Radiat Oncol Biol Phys 2013; 85: 965-970.

9. Cardoso MJ, Oliveira H, Cardoso J. Assessing cosmetic results after breast conserving surgery: The Evolution of Cosmetic Evaluation. J Surg Oncol 2014; 110: 37-44.

10. Harris JR, Levene MB, Svensson G et al. Analysis of cosmetic results following primary radiation therapy for stages I and II carcinoma of the breast. Int J Radiat Oncol Biol Phys 1979; 5: 257-261.

11. Pezner RD, Patterson MP, Hill LR et al. Breast retraction assessment: an objective evaluation of cosmetic results of patients treated conservatively for breast cancer. Int J Radiat Oncol Biol Phys 1985; 11: 575-578.

12. Cardoso MJ, Magalhães A, Almeida T et al. Is face-only photographic view enough for the aesthetic evaluation of breast cancer conservative treatment? Breast Cancer Res Treat 2008 112: 565-568

13. Fitzal F, Mittlboeck M, Trischler $\mathrm{H}$ et al. Breast-conserving therapy for centrally located breast cancer. Ann Surg 2008; 247: 470-476.

14. Ozmen T, Polat AV, Polat AK et al. Factors affecting cosmesis after breast conserving surgery without oncoplastic techniques in an experienced comprehensive breast center. Surgeon 2015; 13: 139-144.

15. Kim MS, Sbalchiero JC, Reece GP et al. Assessment of breast aesthetics. Plast Reconstr Surg 2008; 121: 186e-194e.

16. Munshi A, Kakkar S, Bhutani R et al. Factors influencing cosmetic outcome in breast conservation. Clin Oncol 2009; 21: 285-293.

17. Taylor ME, Perez CA, Halverson KJ et al. Factors influencing cosmetic results after conservation therapy for breast cancer. Int J Radiat Oncol Biol Phys 1995; 31: 753-764.

18. Fitzal F, Krois W, Trischler $\mathrm{H}$ et al. The use of a breast symmetry index for objective evaluation of breast cosmesis. Breast 2007; 16: 429-435.
19. Cardoso MJ, Cardoso J, Amaral N et al. Turning subjective into objective: The BCCT.core software for evaluation of cosmetic results in breast cancer conservative treatment. Breast 2007; 16: 456-461.

20. Yu T, Eom KY, Jang NY et al. Objective measurement of cosmetic outcomes of breast conserving therapy using BCCT. core. Cancer Res Treat 2016; 48: 491-498.

21. Merie R, Browne L, Cardoso JS et al. Proposal for a gold standard for cosmetic evaluation after breast conserving therapy: Results from the St George and Wollongong Breast Boost trial. J Med Imaging Radiat Oncol 2017; 61: 819-825.

22. Gurram L, Wadasadawala T, Joshi K et al. Multi-catheter interstitial brachytherapy for partial breast irradiation: an audit of implant quality based on dosimetric evaluation comparing intra-operative versus post-operative placement. J Contemp Brachytherapy 2016; 8: 116-121.

23. Fayers PM, Aaronson NK, Bjordal $\mathrm{K}$ et al., on behalf of the EORTC Quality of Life Group. The EORTC QLQ-C30 Scoring Manual (3rd ed.). Published by: European Organisation for Research and Treatment of Cancer, Brussels 2001.

24. Didier F, Radice D, Gandini S et al. Does nipple preservation in mastectomy improve satisfaction with cosmetic results, psychological adjustment, body image and sexuality? Breast Cancer Res Treat 2009; 118: 623-633.

25. Aaronson NK, Bartelink H, van Dongen JA et al. Evaluation of breast conserving therapy: clinical, methodological and psychosocial perspectives. Eur J Surg Oncol 1988; 14: 133-140.

26. Christie D, Sharpley C, Curtis T. Improving the accuracy of a photographic assessment system for breast cosmesis. Clin Oncol 2005; 17: 27-31.

27. Olivotto IA, Whelan TJ, Parpia $S$ et al. Interim cosmetic and toxicity results from RAPID: a randomized trial of accelerated partial breast irradiation using three-dimensional conformal external beam radiation therapy. J Clin Oncol 2013; 31: 4038-4045.

28. Bhattacharya IS, Haviland JS, Kirby AM et al. Patient-reported outcomes over 5 years after whole- or partial-breast radiotherapy: longitudinal analysis of the IMPORT LOW (CRUK/06/003) phase III randomized controlled trial. J Clin Oncol 2019; 37: 305-317.

29. Meattini I, Saieva C, Miccinesi G et al. Accelerated partial breast irradiation using intensity modulated radiotherapy versus whole breast irradiation: Health-related quality of life final analysis from the Florence phase 3 trial. Eur J Cancer 2017; 76: 17-26

30. Schäfer R, Strnad V, Polgár C et al. Quality-of-life results for accelerated partial breast irradiation with interstitial brachytherapy versus whole-breast irradiation in early breast cancer after breast-conserving surgery (GEC-ESTRO): 5 -year results of a randomised, phase 3 trial. Lancet Oncol 2018; 19: 834-844.

31. Corica T, Nowak AK, Saunders CM et al. Cosmetic outcome as rated by patients, doctors, nurses and BCCT.core software assessed over 5 years in a subset of patients in the TARGIT-A Trial. Radiat Oncol 2018; 13: 68.

32. Cardoso MJ, Cardoso JS, Oliveira HP et al. The breast cancer conservative treatment. Cosmetic results - BCCT.core - Software for objective assessment of esthetic outcome in breast cancer conservative treatment: A narrative review. Comput Methods Programs Biomed 2016; 126: 154-159.

33. Brouwers PJAM, van Werkhoven E, Bartelink H et al. Predictors for poor cosmetic outcome in early-stage breast cancer treated with breast-conserving therapy: results of the Young boost trial. Radiother Oncol 2018; 128: 434-441.

34. Lagendijk M, Vos EL, Koning AHJ et al. TUmor-volume to breast-volume RAtio for improving COSmetic results in breast cancer patients (TURACOS); a randomized controlled trial. BMC Cancer 2017; 17: 336. 
35. Strnad V, Major T, Polgar C et al. ESTRO-ACROP guideline: Interstitial multi-catheter breast brachytherapy as Accelerated Partial Breast Irradiation alone or as boost - GEC-ESTRO Breast Cancer Working Group practical recommendations. Radiother Oncol 2018; 128: 411-420.

36. Sneeuw KCA, Aaronson NK, Yarnold JR et al. Cosmetic and functional outcomes of breast conserving treatment for early stage breast cancer. 2. Relationship with psychosocial functioning. Radiother Oncol 1992; 25: 160-166.

37. Nahabedian MY, Tsangaris TN. Breast reconstruction following subcutaneous mastectomy for cancer: a critical appraisal of the nipple-areola complex. Plast Reconstr Surg 2006; 117: 1083-1090.

38. Grønkjær M, Eliasen M, Skov-Ettrup LS et al. Preoperative smoking status and postoperative complications: a systematic review and meta-analysis. Ann Surg 2014; 259: 52-71.

39. Niwinska A, Galecki J, Nagadowska M et al. Risk factors of cosmetic outcome in early breast cancer patients after breast conserving therapy. Open Breast Cancer J 2009; 1: 18-24 [cited 2018 Oct 3]. Available from: https:// benthamopen.com/ ABSTRACT/TOBCANJ-1-18

40. Haffty BG, Vicini FA, Beitsch P et al. Timing of chemotherapy after MammoSite radiation therapy system breast brachytherapy: analysis of the American Society of Breast Surgeons MammoSite breast brachytherapy registry trial. Int J Radiat Oncol Biol Phys 2008; 72: 1441-1448.

41. Budrukkar A, Pandit P, Wadasadawala T et al. Impact of adjuvant systemic chemotherapy on wound healing and cosmetic outcome in 224 women treated with accelerated partial breast irradiation using interstitial brachytherapy. Brachytherapy 2017; 16: 935-942. 